\title{
Not-So-Triyne Route to Heteroarylene and Arylene Macrocycles
}

\section{Key words}

electronic materials

[2+2+2]

cycloaddition

rhodium catalysis

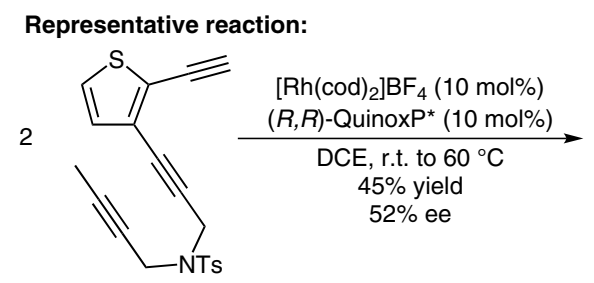

Selected compounds:
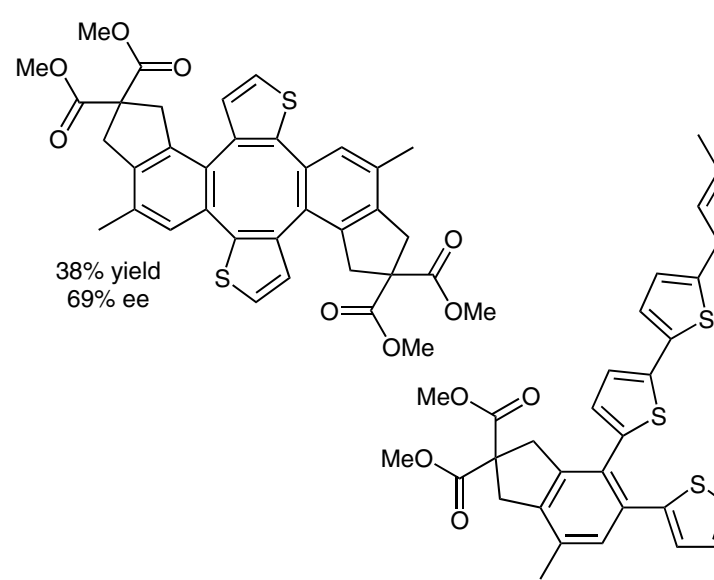

$50 \%$ yield

Significance: This is the first reported synthesis of macrocyclic $\pi$-conjugated structures featuring both benzene and heterocycle units. Isolated yields up to $~ 85 \%$ and stereochemical purity up to $\sim 95 \%$ ee are reported, though it is difficult to achieve both simultaneously. Bis- and tri-thiophene substrates produce trimers. While absorbance maxima were red-shifted with increasing thiophene rings, fluorescence maxima were not, indicating poor conjugation through the benzene ring vertices. However, this resulted in large Stokes shifts (90-120 nm) for these structures.
Comment: Ligand selection was found to be crucial for stereoselectivity, with QuinoxP* overall performing well. In trimer-forming systems, preference for dimer or trimer can also be tuned by ligand choice. The proposed mechanism is two rounds of metallacyclopentadiene formation followed by $[2+2+2]$ cyclization. Stereochemistry is determined by the second [2+2+2] cyclization step. While thiophene-based systems performed well, attempts to use furans resulted in poor yields and stereoselectivity. 